\title{
Régime DASH et risque de cancer colorectal chez les adultes canadiens
}

\author{
E. Jones-Mclean, M. Sc. (1); J. Hu, M.D. (1); L. S. Greene-Finestone, Ph. D. (1, 2); M. de Groh, Ph. D. (1)
}

Cet article a fait l'objet d'une évaluation par les pairs.

Diffuser cet article sur Twitter

\section{Résumé}

Introduction : Le cancer colorectal (CCR) est un cancer à taux d'incidence élevé qui touche de nombreux adultes canadiens chaque année. L'alimentation joue un rôle important dans l'étiologie du CCR et de nombreuses composantes alimentaires sont considérées comme des facteurs de risque potentiels. L'utilisation du profil DASH (Dietary Approaches to Stop Hypertension, régime alimentaire qui vise à lutter contre l'hypertension) est un moyen efficace de caractériser l'alimentation générale des individus. L'objectif de cette étude a été de déterminer un profil DASH en contexte canadien et de vérifier ses liens avec le risque de CCR chez les adultes canadiens.

Méthodologie : Nous avons effectué une régression logistique multiple inconditionnelle avec contrôle des variables de confusion en utilisant des données de l'Étude nationale de surveillance accrue du cancer. Dans cette étude cas-témoins, l'information sur l'alimentation a été recueillie au moyen d'un questionnaire de fréquence de consommation des aliments (QFCA) et un score de 0 à 10 correspondant au profil DASH a été attribué, le score de 0 représentant le profil DASH le plus faible et le score de 10 , le profil DASH le plus fort.

Résultats : Les profils DASH forts (score de 8 ou plus) n'étaient pas fréquents parmi les 3161 cas et les 3097 témoins : dans l'ensemble, seuls 10,8\% des hommes et 13,6 $\%$ des femmes avaient un profil DASH fort. L'analyse multivariée a révélé une tendance à la baisse du risque de CCR chez les hommes avec l'augmentation du score DASH (valeur $p$ de la tendance : 0,007) : chez les hommes à score DASH élevé, le risque de CCR était de $33 \%$ moindre que chez les hommes à score DASH faible. Chez les femmes, il n'y avait aucune tendance statistiquement significative en ce qui concerne le risque de CCR, ou le risque de cancer du côlon ou de cancer du rectum séparément.

Conclusion : Nos résultats sont similaires à ceux d'autres chercheurs et laissent entendre qu'un profil DASH fort aurait un effet bénéfique associé à une diminution du risque de CCR, en particulier chez les hommes. Des travaux de recherche ultérieurs devraient viser à élucider les différences que nous avons observées entre les sexes.

Mots-clés : régime, néoplasie colorectale, prévention primaire

\section{Introduction}

Le cancer colorectal (CCR) est la deuxième cause de mortalité par cancer au Canada, et on prévoyait qu'en 2013, 5000 hommes et 4200 femmes allaient en mourir ${ }^{1}$. Les facteurs de risque de CCR sont les antécédents familiaux, certains syndromes génétiques (p. ex. polypose recto-colique familiale), certaines affections (p. ex. maladies inflammatoires), certains médicaments, certains modes de vie associés à une surcharge pondérale (p. ex. sédentarité) et l'alimentation ${ }^{2}$. On croit que les facteurs alimentaires modifiables jouent un rôle déterminant dans l'étiologie du $\mathrm{CCR}^{3}$.

Il est possible d'étudier les relations entre l'alimentation et certaines maladies chroniques complexes comme le CCR en examinant le régime alimentaire des individus. Les maladies chroniques résultent vraisemblablement de l'accumulation des effets, synergiques ou antagonistes, de diverses composantes alimentaires au fil du temps. Décrire l'exposition alimentaire des individus de façon exhaustive et juste serait donc possible en s'intéressant aux diverses associations d'aliments ou de constituants alimentaires et en les exprimant sous forme d'une mesure synthétique. Les régimes alimentaires les plus répandus sont le régime occidental, le régime dit " prudent » et le régime méditerranéen, mais la liste continue de s'allonger ${ }^{4}$.

Le régime alimentaire DASH (Dietary Approaches to Stop Hypertension), qui vise à lutter contre l'hypertension, favorise une alimentation riche en fruits, en légumes, en céréales entières, en produits laitiers à faible teneur en matières grasses et en légumineuses/graines et pauvre en graisses saturées, en sodium et en sucres ajoutés ${ }^{5}$. C'est dans le but d'entraîner une diminution de la pression artérielle que le régime DASH a été élaboré puis évalué ${ }^{6}$, mais on étudie aujourd'hui ses effets dans d'autres contextes, comme les maladies cardiovasculaires, la fonction rénale, le

Rattachement des auteurs :

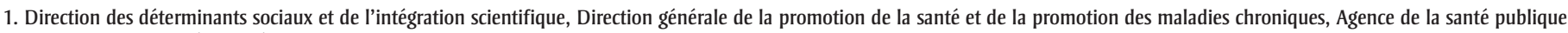
du Canada, Ottawa (Ontario), Canada

2. Division de médecine physique et de réadaptation, Faculté de médecine, Université d'Ottawa, Ottawa (Ontario), Canada

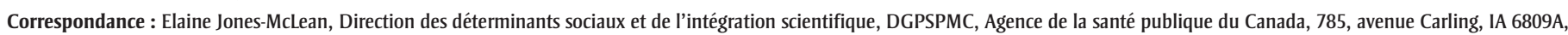
n॰926A2, Ottawa (Ontario) K1A 0K9; tél. : 613-960-6974; téléc. : 613-960-0921; courriel : Elaine.Jones-McLean@phac-aspc.gc.ca 
syndrome métabolique et le diabète gestationnel ${ }^{7-9}$.

Peu d'études ont porté sur la relation entre le régime $\mathrm{DASH}$ et le CCR, et ce, malgré le fait que bon nombre des aliments ou nutriments recommandés dans le régime DASH soient associés à une diminution du risque de $\mathrm{CCR}^{10}$. Les études de Dixon et collab. ${ }^{11}$ et de Fung et collab. ${ }^{12}$, de même qu'une étude sur la fréquence de consommation des aliments utilisant le profil $\mathrm{DASH}^{13}$, ont utilisé des méthodologies différentes pour définir le profil DASH des sujets. Étant donné les différences possibles entre pays quant aux choix alimentaires des individus, nous avons entrepris d'élaborer un profil DASH applicable au contexte canadien pour déterminer ensuite si le fait de suivre un régime DASH était associé à une diminution du risque de CCR. Nous sommes partis de l'hypothèse que plus le score DASH d'un adulte canadien serait élevé, plus son risque de CCR serait faible.

Nous avons choisi le régime DASH plutôt qu'un autre parce que nous croyons que de nombreux Canadiens suivent déjà ce régime pour prévenir ou traiter l'hypertension. Au Canada, la prévalence de l'hypertension est élevée : en 2010, 17,1 \% des Canadiens de 12 ans ou plus avaient reçu un diagnostic d'hypertension artérielle, cette proportion étant considérablement supérieure (40\%) chez les personnes de 65 ans ou plus ${ }^{14}$.

\section{Méthodologie}

Dans le cadre de l'Étude nationale de surveillance accrue du cancer (ENSAC), on a recueilli, entre 1994 et 1997, des données auprès d'un échantillon de population englobant des personnes atteintes de 19 types de cancer. Les cas et les témoins vivaient dans les provinces canadiennes de la Colombie-Britannique, de l'Alberta, de la Saskatchewan, du Manitoba, de l'île-duPrince-Édouard, de la Nouvelle-Écosse et de Terre-Neuve-et-Labrador. Nous avons exclu l'Ontario de nos analyses, car la province n'avait fourni que des témoins, c'est-à-dire aucun cas.

Les résultats de l'ENSAC et d'autres analyses fondées sur le régime alimentaire sont disponibles dans d'autres sources ${ }^{15-17}$.
Les registres des cancers des provinces participantes ont permis de recenser 5112 (2 227 femmes et 2885 hommes) cas incidents de CCR histologiquement confirmé, âgés de 20 à 76 ans. Parmi ces personnes, 325 (6,4 \%; 111 femmes et 214 hommes) étaient déjà décédés au moment où leur médecin a tenté de les joindre, et $341(6,7 \% ; 177$ femmes et 164 hommes) n'ont pu être joints parce que leur médecin traitant n’avait pas donné son consentement (en général parce que les patients étaient trop malades). Sur 4446 questionnaires envoyés par les registres provinciaux des cancers, 3174 ont été remplis, ce qui correspond à un taux de réponse de 62,1 \% des cas recensés ou de $71,4 \%$ des patients ayant été joints. Nous avons vérifié la conformité des cas aux définitions de la Classification Internationale des Maladies pour l'Oncologie (CIM-O-2) ${ }^{18}$, et trois cas (un homme et deux femmes) ont été exclus parce qu'on ne disposait pas des codes correspondants de la CIM-O. Notre analyse a donc porté sur les cas restants, soit un échantillon final de 1816 hommes et de 1355 femmes.

\section{Témoins}

Nous avons choisi des personnes n'ayant pas le cancer à partir d'un échantillon aléatoire pour chaque province participante, avec une répartition par âge et par sexe semblable à celle des cas de cancer de l'ENSAC. En choisissant les témoins, nous nous sommes assurés qu'il y aurait au moins un témoin de même sexe pour chaque cas au sein de chaque groupe d'âge de 5 ans et pour chaque type de cancer. La stratégie d'échantillonnage pour les témoins a été déterminée pour chaque province en fonction de l'expérience de recherche avec diverses bases de données, en fonction de l'accessibilité et de la qualité des données et en fonction des conditions relatives à la confidentialité des bases de données. Elle a donc été différente d'une province à l'autre. Les données du régime provincial d'assurancemaladie ont été utilisées dans le cas de la Colombie-Britannique, de la Saskatchewan, du Manitoba, de l'Île-du-Prince-Édouard et de la Nouvelle-Écosse. En ce qui concerne les témoins de l'Ontario, les données ont été tirées de la base de données d'évaluation foncière du ministère des Finances de l'Ontario. Dans le cas des témoins de l'Alberta et de Terre-Neuve-et-Labrador, on a eu recours à un système d'appel aléatoire. Nous avons par ailleurs échelonné le recrutement des témoins sur une année entière, de façon à assurer une distribution uniforme des réponses, qui peuvent varier en fonction de la saison ( $p$. ex. quant à l'alimentation et à la pratique d'une activité physique). En Ontario, une petite somme d'argent a été offerte en guise d'incitatif, afin d'obtenir un meilleur taux de réponse.

Sur les 5119 questionnaires envoyés à des témoins potentiels, 81 ont été retournés en raison d'une erreur dans l'adresse et, parmi les questionnaires restants, 3097 (1 635 hommes et 1462 femmes) ont été remplis, ce qui représente un taux de réponse de $61,5 \%$ des personnes jointes ${ }^{19-20}$.

\section{Collecte des données}

L'inscription des cas aux registres provinciaux des cancers a été faite habituellement dans les 1 à 3 mois suivant le diagnostic, au moyen des rapports d'anatomopathologie. Après avoir reçu le consentement des médecins, les responsables des registres ont envoyé un questionnaire aux participants potentiels (cas et témoins). Lorsqu'un questionnaire n'a pas été retourné au bout de deux semaines, une carte de rappel a été envoyée et, au bout de 4 semaines, un autre exemplaire du questionnaire a été envoyé. Après 6 semaines, les personnes n'ayant pas retourné leur questionnaire ont reçu un rappel téléphonique. Le protocole utilisé pour la collecte des données auprès des témoins a été le même que celui utilisé auprès des cas.

Les données recueillies ont concerné le statut socioéconomique, la taille et le poids autodéclarés, les antécédents tabagiques, la consommation d'alcool, la pratique d'activité physique, les antécédents menstruels et génésiques et l'alimentation.

Dans le cas du poids autodéclaré, on a demandé aux participants de se rappeler leur poids 2 ans avant l'étude, afin de calculer leur indice de masse corporelle (IMC, en $\left.\mathrm{kg} / \mathrm{m}^{2}\right)^{21}$. 
En ce qui concerne le tabagisme, la catégorie des "sujets ayant déjà fumé " englobait les personnes qui avaient fumé au moins 100 cigarettes au cours de leur vie et la catégorie des "fumeurs actuels » englobait les personnes qui fumaient encore dans les 12 mois ayant précédé l'enquête.

Pour obtenir l'information sur la pratique d'une activité physique durant les loisirs, on a demandé aux participants combien de temps ils avaient consacré à la pratique d'une activité physique modérée et d'une activité physique intense au cours des 24 mois ayant précédé l'étude.

Nous avons obtenu l'information sur l'alimentation des participants en utilisant un questionnaire semi-quantitatif sur la fréquence de consommation des aliments (QFCA) fondé sur deux instruments validés : le questionnaire abrégé de Block $^{22}$ et le questionnaire de Willett $^{23}$. Nous avons utilisé le QFCA pour déterminer l'apport alimentaire habituel des sujets 2 ans avant leur participation à l'étude. Le QFCA portait sur 69 aliments et boissons précis, répartis en huit groupes : a) pains et céréales, b) viande, volaille, poisson, œufs et fromage, c) légumes, d) fruits, e) sucreries, f) aliments divers, comme le beurre d'arachides et les noix, g) boissons à base d'eau, comme le café, le thé, les jus, etc., h) autres boissons, comme les boissons gazeuses, le lait et les boissons alcoolisées. Pour chaque aliment ou boisson, les participants devaient décrire à quelle fréquence (par jour, par semaine, par mois), en moyenne, ils consommaient la portion indiquée. Nous avons utilisé une base de données fondée sur le Fichier canadien sur les éléments nutritifs pour estimer la consommation d'éléments nutritifs et l'apport énergétique total en fonction du profil nutritionnel des aliments à ce moment-là ${ }^{24}$.

Nous avons utilisé une échelle à 10 points pour décrire le profil DASH des participants (voir le tableau 1) plutôt que d'uti- liser une échelle à 9 points comme cela a été fait dans certaines études ${ }^{11,25}$. Nous avons élaboré notre échelle de scores en fonction des aliments ou groupes alimentaires mentionnés dans le plan pour une alimentation conforme au régime DASH des lignes directrices américaines sur l'alimentation $^{26}$ et dans une publication connexe $^{11}$ pour tenir compte de la consommation de céréales entières, de légumes, de fruits, de produits laitiers à faible teneur en matières grasses, de viandes rouges et transformées, de sucreries, d'alcool, de graisses saturées et de noix, légumineuses et graines. Nous avons modifié notre échelle en y ajoutant un dixième point, soit l'apport en sodium, comme l'ont fait d'autres chercheurs ${ }^{12}$.

Pour mesurer l'apport énergétique total, nous avons établi des quartiles en fonction de la distribution de l'apport énergétique chez les témoins. Pour chaque quartile, nous avons calculé l'apport médian pour chacune des 10 catégories alimentaires en

TABLEAU 1

Schéma pour le calcul du score DASH

Catégories alimentaires
POSITIVES
Céréales entières
Légumes
Fruits
Produits laitiers à faible teneur en matières grasses
Noix/graines/légumineuses

NÉGATIVES

Viande (rouge, transformée)

Graisses saturées

Sodium

Alcool

Sucreries

\section{Exemples d'aliments (ou de calculs) pour le QFCA}

\section{1 point pour les apports supérieurs ou égaux à la médiane;}

0 point pour les apports inférieurs à la médiane
Son, mélange céréalier, blé filamenté, céréales cuites, pain noir et pain de blé entier

Tomates, carottes, brocoli, chou, chou-fleur, choux de Bruxelles, épinards ou autres légumes-feuilles, potirons, patates douces, haricots verts, maïs, pois, etc.

Pommes, poires, oranges, bananes, cantaloups, autres fruits frais ou en conserve, jus d'orange ou de pamplemousse

Lait $2 \%$, lait $1 \%$, lait écrémé

Noix, tofu, soja, fèves au lard, lentilles
Pain blanc, riz, macaroni

Frites, soupe aux légumes ou aux tomates, jus de légumes

Aliments avec sucre ajouté, comme les boissons à base de concentré ou les boissons en poudre

Lait entier, fromage ordinaire, crème glacée Beurre d'arachides à forte teneur en matières grasses

1 point pour les apports inférieurs ou égaux à la médiane; 0 point pour les apports supérieurs à la médiane

Bouf, porc, agneau (y compris les mélanges), Poisson, volaille, oufs hamburger, saucisses, hot-dog, bœuf mariné ou salé, viandes froides, foie

Apport total en graisses saturées pour l'ensemble des aliments visés, selon la formule suivante : $\%=$ graisses saturées $(\mathrm{g}) \times 9(\mathrm{kcal}) /$ apport énergétique alimentaire total (kcal)

Apport total en sodium pour l'ensemble des aliments visés

Bière, vin, spiritueux

Gâteaux, biscuits, beignes, pâtisseries, tartes, crème glacée, chocolat, boissons gazeuses, boissons en poudre, etc. 
utilisant l'apport des témoins, après stratification par sexe. Les quartiles d'apport énergétique que nous avons utilisés étaient les suivants : $1458 \mathrm{kcal} /$ jour ou moins, de 1459 à $1843 \mathrm{kcal} /$ jour, de 1844 à $2284 \mathrm{kcal} / \mathrm{jour}$, et $2285 \mathrm{kcal} / \mathrm{jour}$ ou plus. Les participants recevaient un point pour un apport énergétique égal ou supérieur à la médiane des sujets du même sexe pour les catégories alimentaires «positives » suivantes : céréales entières, légumes, fruits, produits laitiers à faible teneur en matières grasses et légumineuses/noix/graines. Les apports inférieurs à la médiane pour ces catégories alimentaires entraînaient l'attribution d'un score de zéro. De même, les participants recevaient un point pour un apport égal ou inférieur à la médiane pour les catégories alimentaires "négatives " suivantes : viandes rouges et transformées, graisses saturées, alcool et sucreries. Dans le cas de ces catégories alimentaires, les apports supérieurs à la médiane entraînaient l'attribution d'un score de zéro.

Nous avons réparti les aliments visés par le QFCA dans les groupes appropriés et nous avons calculé le nombre de portions de chaque aliment en nous fondant sur la méthodologie employée pour le régime $\mathrm{DASH}^{5,11,26}$. Lorsqu'il nous manquait des données, nous complétions cette approche en examinant des nutriments communs à d'autres aliments d'un même groupe afin de trouver un équivalent approprié. Cela s'est avéré particulièrement utile pour les groupes englobant des aliments hétérogènes, comme les sucreries. Comme les données sur la teneur en sucres des aliments sont limitées dans le Fichier canadien sur les éléments nutritifs ${ }^{24}$, nous avons évalué les aliments du groupe des sucreries en fonction des calories. Ainsi, un biscuit équivalait à une portion (54 kcal), et un verre de boisson gazeuse équivalait à deux portions (98 kcal). Pour ce qui est de l'apport en graisses saturées et en sodium, nous ne nous sommes pas fiés à la consommation d'aliments précis comme nous l'avons fait avec les autres groupes alimentaires visés par le QFCA : nous avons plutôt attribué un score aux participants en déterminant l'apport total en ces nutriments à partir de l'ensemble des aliments consommés et en effectuant une comparaison avec la médiane de l'apport total pour l'ensemble des quartiles.

Le score DASH a été calculé sur une échelle de 0 à 10. Dans notre étude, les scores DASH représentent un profil représentatif du régime DASH car ils sont fondés sur des estimations supérieures ou inférieures aux médianes spécifiques du sexe et de l'apport énergétique. Par conséquent, un score DASH de 8 ou plus est considéré comme un profil «fort », et un score de 2 ou moins est considéré comme un profil « faible ».

\section{Analyse statistique}

Nous avons effectué une régression logistique inconditionnelle stratifiée en fonction du sexe pour estimer les rapports de cotes (RC) et les intervalles de confiance (IC) à $95 \%$ correspondants en utilisant des termes pour les groupes d'âge (20 à 49 ans, 50 à 59 ans, 60 à 69 ans, 70 ans et plus), pour la province, pour le niveau de scolarité (8 ans ou moins, 9 à 13 ans, 14 ans ou plus), pour l'IMC (moins de $25,0 \mathrm{~kg} / \mathrm{m}^{2}, 25,0$ à $29,9 \mathrm{~kg} / \mathrm{m}^{2}, 30,0 \mathrm{~kg} / \mathrm{m}^{2}$ ou plus), pour le tabagisme (paquetsannées), pour le revenu, pour la pratique d'une activité physique modérée et intense durant les loisirs, pour la prise de suppléments de calcium et pour l'âge à la première grossesse. À l'exception du groupe d'âge, de la province, de l'IMC et du sexe, les variables de confusion ont été traitées comme des variables continues dans les modèles. Nous avons effectué des tests de tendance pour chaque variable à l'étude, en donnant aux variables du modèle une forme continue.

Les analyses ont été effectuées à l'aide de la version $9.01 \mathrm{du}$ progiciel de statistiques SAS (SAS Institute Inc., Cary [Caroline du Nord], États-Unis) ${ }^{27}$.

\section{Résultats}

Les participants à l'étude étaient 3171 pour les cas et 3097 pour les témoins, les hommes (3 451) étant $23 \%$ plus nombreux que les femmes (2 817). La plupart des participants avaient un niveau de scolarité supérieur ou égal aux études secondaires, avaient un revenu familial modéré à élevé et avaient déjà fumé ou étaient fumeurs. Les cas étaient généralement des sujets plus âgés présentant un IMC élevé, et les femmes atteintes de CCR ayant eu un enfant l'avaient généralement eu après l'âge de 20 ans. Parmi les participants ayant déclaré leur revenu familial, il n'y avait aucune différence statistiquement significative entre les cas et les témoins (tableau 2).

L'apport médian associé aux aliments et aux nutriments avait tendance à augmenter avec l'augmentation de l'apport énergétique. L'alcool faisait figure d'exception, l'apport associé à cette substance demeurant relativement stable chez les femmes dans l'ensemble des quartiles d'apport énergétique (tableau 3). L'apport en graisses saturées était semblable chez les hommes et les femmes dans l'ensemble des quartiles d'apport énergétique, oscillant entre $1,5 \%$ et $1,7 \%$ de l'apport énergétique total.

Les profils DASH forts (score de 8 ou plus) n'étaient pas courants chez les participants à l'étude (tableau 4) : dans l'ensemble, seuls 10,8 \% des hommes (374/3 451) et $13,6 \%$ des femmes (382/ 2 817) ont obtenu un score de 8 ou plus (tableau 4). De façon analogue, seul un faible pourcentage de participants ont obtenu un score DASH faible (2 ou moins), dénotant une faible correspondance entre leurs habitudes alimentaires et le régime DASH : 10,1\% des hommes (349/3 451) et 10,2 \% des femmes (286/ 2 817). Environ $50 \%$ des participants ont obtenu un score DASH modéré, soit variant entre 4 et 6 .

Nos analyses ont révélé une tendance marquée à la baisse du risque de CCR avec l'augmentation du score DASH (valeur $p$ de la tendance : 0,007) chez les hommes. Après ajustement en fonction des variables de confusion, le risque de CCR était de $33 \%$ moindre chez les hommes ayant obtenu un score de 8 ou plus que chez ceux ayant obtenu un score inférieur. Chez les hommes, il y avait une tendance à la baisse du risque de cancer du rectum ( $p=0,003)$ avec l'augmentation du score DASH, mais pas du risque de cancer du côlon ( $p=0,09)$, bien qu'une tendance similaire ait été visible. Chez les 
TABLEAU 2

Distribution des cas de cancer colorectal $(3$ 171) et des témoins tirés de la population (3 097) en fonction de diverses covariables, ENSAC, Canada, 1994-1997

\begin{tabular}{|c|c|c|c|c|c|}
\hline & \multicolumn{2}{|c|}{ Cas } & \multicolumn{2}{|c|}{ Témoins } & \multirow{2}{*}{$\begin{array}{c}\text { Valeur } \boldsymbol{p} \text { associée } \\
\text { au chi carré }\end{array}$} \\
\hline & $\mathbf{n}$ & $\%$ & $\mathbf{N}$ & $\%$ & \\
\hline \multicolumn{6}{|l|}{ Sexe } \\
\hline Hommes & 1816 & 57,2 & 1635 & 52,8 & \\
\hline Femmes & 1355 & 42,8 & 1462 & 47,2 & \\
\hline \multicolumn{6}{|l|}{ Âge (ans) } \\
\hline 20 à 49 & 378 & 11,9 & 838 & 27,1 & $<0,0001$ \\
\hline 50 à 59 & 645 & 20,4 & 605 & 19,5 & \\
\hline 60 à 69 & 1342 & 42,3 & 1043 & 33,7 & \\
\hline 70 et plus & 806 & 25,4 & 611 & 19,7 & \\
\hline \multicolumn{6}{|l|}{ Niveau de scolarité (ans) } \\
\hline 8 ou moins & 577 & 18,2 & 471 & 15,2 & $<0,0001$ \\
\hline 9 à 13 & 1818 & 57,3 & 1689 & 54,5 & \\
\hline 14 ou plus & 711 & 22,4 & 900 & 29,1 & \\
\hline Valeurs manquantes & 65 & 2,1 & 37 & 1,2 & \\
\hline \multicolumn{6}{|l|}{ Revenu familial $^{a}$} \\
\hline Faible & 584 & 18,4 & 584 & 18,9 & 0,32 \\
\hline Moyen inférieur & 570 & 18,0 & 585 & 18,9 & \\
\hline Moyen supérieur & 758 & 23,9 & 779 & 25,2 & \\
\hline Élevé & 474 & 14,9 & 440 & 14,2 & \\
\hline Valeurs manquantes & 785 & 24,8 & 709 & 22,9 & \\
\hline \multicolumn{6}{|l|}{ Tabagisme (paquets-années) } \\
\hline Sujets n'ayant jamais fumé & 995 & 31,4 & 1123 & 36,5 & $<0,0001$ \\
\hline 10 ou moins & 626 & 19,7 & 705 & 23,0 & \\
\hline 11 à 20 & 525 & 16,6 & 470 & 15,3 & \\
\hline 21 à 30 & 377 & 11,9 & 302 & 9,8 & \\
\hline Plus de 30 & 592 & 18,7 & 447 & 14,5 & \\
\hline Valeurs manquantes & 56 & 1,8 & 50 & 1,6 & \\
\hline \multicolumn{6}{|l|}{ IMC $\left(\mathbf{k g} / \mathbf{m}^{2}\right)$} \\
\hline Moins de 25,0 & 1175 & 37,1 & 1461 & 47,2 & $<0,0001$ \\
\hline 25,0 à 29,9 & 1345 & 42,4 & 1176 & 38,0 & \\
\hline 30,0 et plus & 637 & 20,1 & 447 & 14,4 & \\
\hline Valeurs manquantes & 14 & 0,2 & 13 & 0,4 & \\
\hline \multicolumn{6}{|c|}{ Activité physique modérée (heures/mois) } \\
\hline 4,22 ou moins & 598 & 18,9 & 638 & 20,6 & $<0,0019$ \\
\hline 4,23 à 11,57 & 645 & 20,3 & 702 & 22,7 & \\
\hline 11,58 à 24,44 & 720 & 22,7 & 725 & 23,4 & \\
\hline 24,45 ou plus & 730 & 23,0 & 636 & 20,5 & \\
\hline Valeurs manquantes & 478 & 15,1 & 396 & 12,8 & \\
\hline \multicolumn{6}{|c|}{ Activité physique intense (heures/mois) } \\
\hline Jamais & 1324 & 41,8 & 1146 & 37,0 & $<0,0006$ \\
\hline 0,19 ou moins & 174 & 5,5 & 162 & 5,2 & \\
\hline 0,20 à 3,68 & 565 & 17,8 & 644 & 20,8 & \\
\hline 3,69 ou plus & 597 & 18,8 & 647 & 20,9 & \\
\hline Valeurs manquantes & 511 & 16,1 & 498 & 16,1 & \\
\hline
\end{tabular}

Suite page suivante femmes, il n’y avait aucune tendance statistiquement significative associée à une augmentation du score DASH, que ce soit pour le cancer du côlon, le cancer du rectum ou ces deux cancers combinés.

En stratifiant les analyses en fonction de l'IMC (tableau 5), nous n'avons observé aucune interaction entre le score DASH et le risque de CCR. Chez les hommes non obèses et sans surpoids (IMC inférieur à $25,0 \mathrm{~kg} / \mathrm{m}^{2}$ ), la tendance relative au cancer du rectum $(p=0,01)$ était statistiquement significative, et la tendance relative au CCR ( $p=0,05)$ était au seuil de signification statistique. Chez les hommes à profil DASH fort, le risque de cancer du rectum était réduit de $50 \%$ et le risque de CCR de $36 \%$. Chez les hommes qui présentaient un surpoids ou étaient obèses (IMC $\geq 25,0 \mathrm{~kg} / \mathrm{m}^{2}$ ), le risque de CCR était réduit de $35 \%$ pour ceux à profil DASH fort, mais était toutefois au seuil de signification statistique. Bien que cette tendance n'ait pas été statistiquement significative $(p=0,07)$, il semblait $\mathrm{y}$ avoir une diminution du risque de cancer du rectum chez les hommes présentant un surpoids ou obèses avec l'augmentation du score DASH.

Chez les femmes de tous les groupes d'IMC, il n'y avait aucune association statistiquement significative entre l'augmentation du score DASH et le risque de cancer, tous types confondus.

Nous avons également examiné la parité des femmes afin de déterminer s'il s'agissait d'un facteur de confusion, mais nous n’avons observé aucune différence statistique entre les cas et les témoins (données non présentées).

\section{Analyse}

Cette étude est la première au Canada à porter sur la relation potentielle entre le profil DASH et le risque de CCR.

Nos résultats concordent avec ceux d'autres études ayant révélé l'existence d'une relation inverse entre un profil DASH fort et le risque de CCR, quoiqu'avec une certaine variabilité entre les sexes $^{11-13,28}$. Fung et collab. ${ }^{12}$ ont fait état d'une association protectrice dans le cas 
TABLEAU 2 (Suite)

Distribution des cas de cancer colorectal $(3$ 171) et des témoins tirés de la population (3 097) en fonction de diverses covariables, ENSAC, Canada, 1994-1997

\begin{tabular}{|c|c|c|c|c|c|}
\hline & \multicolumn{2}{|c|}{ Cas } & \multicolumn{2}{|c|}{ Témoins } & \multirow{2}{*}{$\begin{array}{l}\text { Valeur } \boldsymbol{p} \text { associée } \\
\text { au chi carré }\end{array}$} \\
\hline & n & $\%$ & $\mathbf{N}$ & $\%$ & \\
\hline \multicolumn{6}{|c|}{ Suppléments de calcium } \\
\hline Jamais & 1944 & 61,3 & 1849 & 59,7 & $<0,0001$ \\
\hline Pas régulièrement & 603 & 19,0 & 649 & 20,9 & \\
\hline Régulièrement & 369 & 11,6 & 430 & 13,9 & \\
\hline Valeurs manquantes & 255 & 8,0 & 169 & 5,5 & \\
\hline \multicolumn{6}{|c|}{ Âge à la première grossesse (ans) } \\
\hline 20 ou moins & 270 & 19,9 & 358 & 24,5 & $<0,01$ \\
\hline 21 à 23 & 343 & 25,3 & 343 & 23,5 & \\
\hline 24 à 26 & 238 & 17,6 & 239 & 16,4 & \\
\hline Plus de 26 & 302 & 22,3 & 283 & 19,4 & \\
\hline Valeurs manquantes & 202 & 14,9 & 239 & 16,4 & \\
\hline
\end{tabular}

Abréviations : ENSAC, Étude nationale de surveillance accrue du cancer; IMC, indice de masse corporelle.

${ }^{a}$ Le revenu familial figure à titre de variable nominale et est associé aux valeurs suivantes : faible : $<20000 \$$ si 3 personnes ou moins ou < $30000 \$$ si 4 personnes ou plus; moyen inférieur : $20000 \$$ à $30000 \$$ si 3 personnes ou moins ou $30000 \$$ à $50000 \$$ si 4 personnes ou plus; moyen supérieur : < $50000 \$$ si 3 personnes ou moins ou $50000 \$$ à $100000 \$$ si 4 personnes ou plus; élevé : $\geq 50000 \$$ si 3 personnes ou moins ou $\geq 100000 \$$ si 4 personnes ou plus.

du cancer du côlon proximal chez les femmes suivant un régime de type DASH ou méditerranéen, mais non chez les hommes suivant un tel régime. Dans notre étude, le régime DASH s'est révélé protecteur chez les hommes, mais non chez les femmes. Nos résultats concordent avec ceux de Dixon et collab. ${ }^{11}$, qui font état d'une association statistiquement significative entre l'augmentation du score DASH et une diminution du risque d'adé- nomes associés au CCR distal chez les hommes, sans égard à d'autres facteurs, comme le poids ou le tabagisme. D'autres études ont également fait état d'une relation inverse entre le risque de CCR et l'obtention d'un score élevé en lien avec le régime DASH ou à un autre régime alimentaire sain chez les hommes, mais non chez les femmes ${ }^{13,29}$. Certains chercheurs ont expliqué que cette divergence était due au fait que l'étiologie du CCR était différente chez les hommes et chez les femmes ${ }^{29}$.

Selon divers chercheurs, les hommes et les femmes réagissent différemment à une intervention alimentaire ${ }^{30,31}$. Une étude canadienne a révélé que la concentration d'insuline deux heures après l'administration de glucose était meilleure chez les hommes suivant un régime méditerranéen que chez les femmes suivant le même régime ${ }^{30}$. De plus, seuls les participants de sexe masculin ont vu une diminution statistiquement significative de leur IMC en suivant le régime méditerranéen. Ces deux résultats ont été attribués à une meilleure sensibilité à l'insuline et à une meilleure homéostasie chez les hommes ${ }^{30}$.

Dans un autre groupe d'adultes, le régime méditerranéen était associé à une sensibilité accrue à l'insuline chez les hommes jeunes, mais pas chez les femmes en

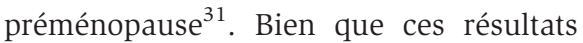
sexospécifiques n’aient pas été évalués dans le contexte du CCR ou d'un autre cancer, la réponse insulinique a d'importantes implications quant au risque de cancer colorectal. L'insuline et le facteur de croissance insulinomimétique de type 1 peuvent favoriser l'apparition du CCR en activant une série de voies de signalisation associées à une augmentation du risque oncogène ${ }^{32}$. Le rôle possible de l'insuline

TABLEAU 3

Apport médian associé aux catégories alimentaires en fonction du sexe et du quartile d'apport énergétique, ENSAC, Canada, 1994-1997

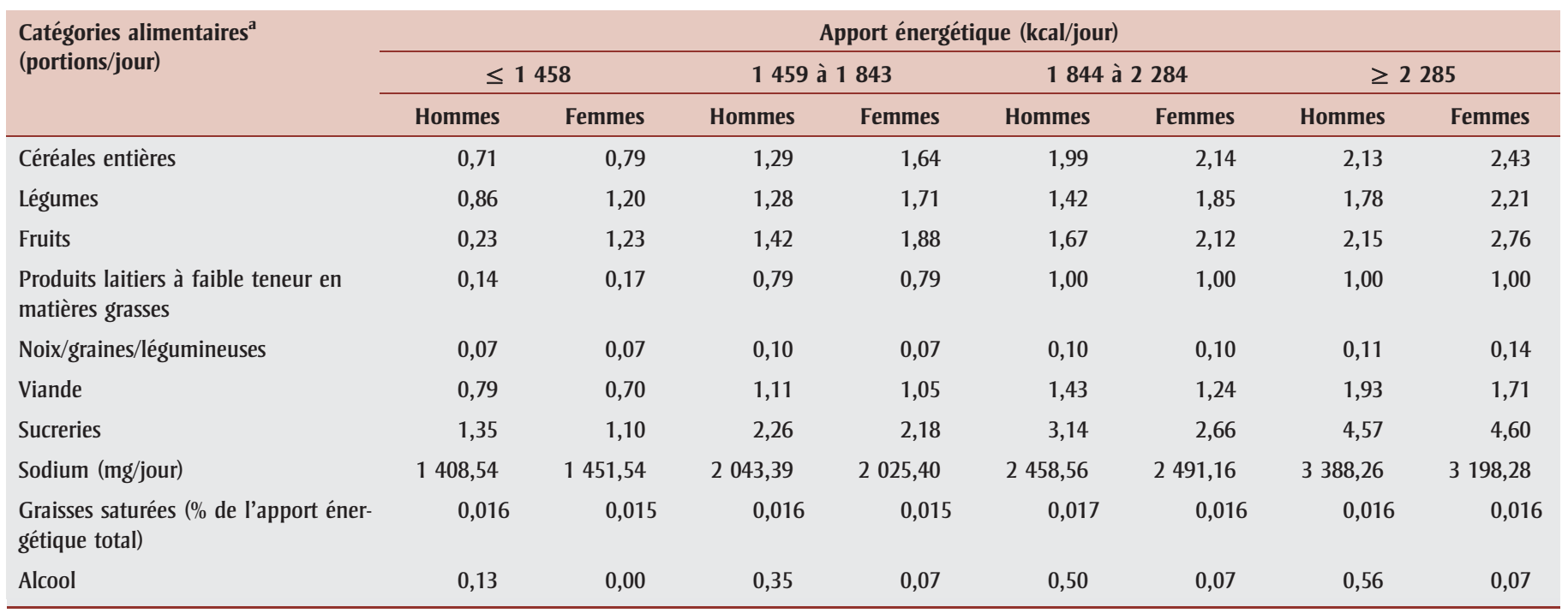

Abréviations : DASH, Dietary Approaches to Stop Hypertension; ENSAC, Étude nationale de surveillance accrue du cancer; IC, intervalle de confiance; RC, rapport de cotes.

${ }^{a}$ Les catégories alimentaires sont les mêmes que dans le tableau 1. 
TABLEAU 4

Rapports de cotes ${ }^{\mathrm{a}}$ et intervalles de confiance à $95 \%$ associés au cancer colorectal selon le score médian, par sexe, ENSAC, Canada, $1994-1997$

\begin{tabular}{|c|c|c|c|c|c|c|c|c|}
\hline \multirow[t]{2}{*}{ Siège du cancer } & \multicolumn{7}{|c|}{ Score DASH } & \multirow{2}{*}{$\begin{array}{c}\text { Valeur } p \\
\text { associée à } \\
\text { la tendance }\end{array}$} \\
\hline & $\leq 2$ & 3 & 4 & 5 & 6 & 7 & $\geq 8$ & \\
\hline \multicolumn{9}{|l|}{ Côlon } \\
\hline \multicolumn{9}{|l|}{ Hommes } \\
\hline Cas & 93 & 124 & 169 & 174 & 177 & 130 & 89 & \\
\hline Témoins & 181 & 226 & 272 & 279 & 242 & 217 & 216 & \\
\hline \multicolumn{9}{|l|}{ Femmes } \\
\hline Cas & 71 & 89 & 135 & 149 & 111 & 99 & 108 & \\
\hline Témoins & 152 & 173 & 259 & 251 & 225 & 202 & 196 & \\
\hline RC (IC à 95 \%) & Réf. & $1,04(0,69-1,57)$ & $1,12(0,76-1,64)$ & $1,06(0,72-1,55)$ & $1,01(0,67-1,51)$ & $1,00(0,66-1,51)$ & $1,15(0,76-1,74)$ & 0,81 \\
\hline \multicolumn{9}{|l|}{ Rectum } \\
\hline RC (IC à $95 \%)$ & Réf. & $1,32(0,91-1,93)$ & $1,57(1,10-2,25)$ & $1,27(0,88-1,83)$ & $1,26(0,87-1,83)$ & $1,01(0,68-1,50)$ & $0,64(0,42-0,98)$ & 0,003 \\
\hline \multicolumn{9}{|l|}{ Femmes } \\
\hline Cas & 63 & 67 & 79 & 112 & 108 & 82 & 78 & \\
\hline Témoins & 152 & 173 & 259 & 251 & 225 & 202 & 196 & \\
\hline RC (IC à 95 \%) & Réf. & $1,02(0,66-1,57)$ & $0,79(0,52-1,19)$ & $0,98(0,65-1,47)$ & $1,23(0,81-1,97)$ & $0,92(0,59-1,42)$ & $1,03(0,66-1,60)$ & 0,58 \\
\hline \multicolumn{9}{|l|}{ Colorectum } \\
\hline \multicolumn{9}{|l|}{ Hommes } \\
\hline Cas & 168 & 252 & 342 & 332 & 320 & 240 & 158 & \\
\hline Témoins & 181 & 226 & 272 & 279 & 242 & 217 & 216 & \\
\hline
\end{tabular}

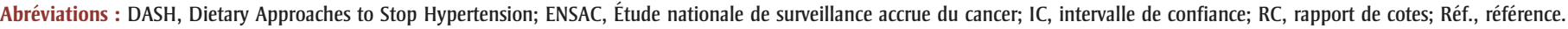

Remarque : Les totaux peuvent varier du fait de valeurs manquantes.

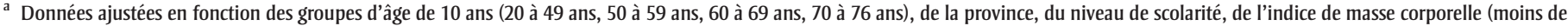
25,0, 25,0 à 29,9, 30,0 ou plus), du nombre de paquets-années dans le cas des fumeurs, de la pratique d'une activité physique modérée ou intense, de la prise de suppléments de calcium et de l'âge à la première grossesse.

dans l'apparition du CCR est corroboré par l'association observée entre le diabète de type 2 et un risque accru de cancer, notamment de $\mathrm{CCR}^{33,34}$. Étant donné que le régime méditerranéen et le régime DASH sont très semblables (ils mettent tous deux l'accent sur la consommation de céréales entières, de noix et de légumineuses et sur une consommation limitée de sucreries) et qu'il existe une étroite corrélation entre ceux-ci ${ }^{12}$, il est possible que nos observations chez les hommes soient uniquement attribuables aux processus métaboliques associés à la sensibilité à l'insuline.
Nous avons stratifié les participants en fonction de leur IMC car il est possible que les habitudes alimentaires influent sur le risque de CCR chez les personnes à risque élevé d'insulinorésistance seulement (celles ayant un IMC élevé) ${ }^{35}$. Cependant, selon nos observations, l'effet protecteur du régime DASH ne se limitait pas aux personnes ayant un surpoids ou aux personnes obèses. Nous avons constaté que les scores DASH élevés étaient associés à un effet protecteur contre le cancer du rectum chez les hommes de poids normal, et que ces scores étaient associés à un effet protecteur au seuil de signification statis- tique dans le cas du CCR chez les hommes de poids normal, les hommes ayant un surpoids et les hommes obèses. Chez les femmes, nous n'avons observé aucune tendance statistique pour ce qui est du cancer du rectum, du cancer du côlon ou de ces deux cancers combinés.

Afin de mieux comprendre la raison pour laquelle l'effet protecteur se manifestait uniquement chez les hommes, nous avons examiné des facteurs liés à la santé génésique. Nous avons évalué la parité des femmes, un facteur potentiellement associé à une diminution du risque de $\mathrm{CCR}^{36-38}$, 
TABLEAU 5

Rapports de $\operatorname{cotes}^{\mathrm{a}}$ et intervalles de confiance à $95 \%$ associés au cancer colorectal selon le score DASH médian, l'indice de masse corporelle et le sexe, ENSAC, Canada, 1994-1997

\begin{tabular}{|c|c|c|c|c|c|c|c|c|}
\hline \multirow[t]{2}{*}{ Siège du cancer } & \multicolumn{7}{|c|}{ Score DASH } & \multirow{2}{*}{$\begin{array}{l}\text { Valeur } p \\
\text { associée à la } \\
\text { tendance }\end{array}$} \\
\hline & $\leq 2$ & 3 & 4 & 5 & 6 & 7 & $\geq 8$ & \\
\hline \multicolumn{9}{|l|}{ IMC $<25,0 \mathrm{~kg} / \mathrm{m}^{2}$} \\
\hline Hommes $(n=274)$ & Réf. & $1,30(0,68-2,51)$ & $0,84(0,45-1,60)$ & $0,86(0,45-1,63)$ & $1,39(0,74-2,62)$ & $0,94(0,48-1,85)$ & $0,69(0,34-1,40)$ & 0,40 \\
\hline Femmes $(n=355)$ & Réf. & $1,53(0,79-2,96)$ & $1,49(0,81-2,73)$ & $1,53(0,82-2,84)$ & $2,09(1,13-3,89)$ & $1,60(0,84-3,05)$ & $1,65(0,86-3,17)$ & 0,16 \\
\hline Hommes $(n=268)$ & Réf. & $1,55(0,80-3,01)$ & $1,34(0,72-2,51)$ & $0,89(0,46-1,72)$ & $1,25(0,64-2,43)$ & $0,91(0,45-1,83)$ & $0,50(0,24-1,07)$ & 0,01 \\
\hline Femmes $(n=278)$ & Réf. & $0,97(0,51-1,84)$ & $0,77(1,43-1,39)$ & $0,88(0,48-1,60)$ & $0,91(0,49-1,69)$ & $0,74(0,39-1,40)$ & $1,04(0,56-1,95)$ & 0,96 \\
\hline \multicolumn{9}{|l|}{ Colorectum ( $n=1$ 175) } \\
\hline Hommes $(n=542)$ & Réf. & $1,48(0,87-2,52)$ & $1,18(0,71-1,95)$ & $0,92(0,55-1,55)$ & $1,40(0,83-2,36)$ & $0,98(0,57-1,70)$ & $0,64(0,36-1,14)$ & 0,05 \\
\hline Femmes $(n=633)$ & Réf. & $1,32(0,79-2,20)$ & $1,10(0,69-1,76)$ & $1,17(0,72-1,89)$ & $1,43(0,88-2,32)$ & $1,13(0,68-1,87)$ & $1,32(0,80-2,19)$ & 0,41 \\
\hline Femmes $(n=403)$ & Réf. & $0,74(0,44-1,24)$ & $1,03(0,63-1,69)$ & $0,74(0,45-1,21)$ & $0,73(0,42-1,25)$ & $0,75(0,44-1,30)$ & $0,78(0,42-1,45)$ & 0,30 \\
\hline \multicolumn{9}{|l|}{ Rectum $(n=891)$} \\
\hline Hommes $(n=586)$ & Réf. & $1,21(0,77-1,91)$ & $1,64(1,05-2,56)$ & $1,45(0,93-2,26)$ & $1,24(0,79-1,96)$ & $1,06(0,66-1,71)$ & $0,70(0,41-1,17)$ & 0,07 \\
\hline Femmes $(n=305)$ & Réf. & $1,02(0,56-1,86)$ & $0,75(0,41-1,38)$ & $1,10(0,63-1,92)$ & $1,58(0,90-2,80)$ & $1,06(0,58-1,93)$ & $0,65(0,50-1,82)$ & 0,47 \\
\hline \multicolumn{9}{|l|}{ Colorectum ( $n$ = 1974$)$} \\
\hline Hommes ( $n=1267)$ & Réf. & $0,99(0,68-1,43)$ & $1,35(0,95-1,94)$ & $1,29(0,91-1,84)$ & $1,18(0,82-1,70)$ & $0,95(0,65-1,39)$ & $0,65(0,43-0,98)$ & 0,05 \\
\hline Femmes $(n=707)$ & Réf. & $0,85(0,53-1,36)$ & $0,88(0,56-1,38)$ & $0,96(0,62-1,48)$ & $0,85(0,54-1,35)$ & $0,83(0,52-1,33)$ & $0,93(0,57-1,52)$ & 0,78 \\
\hline
\end{tabular}

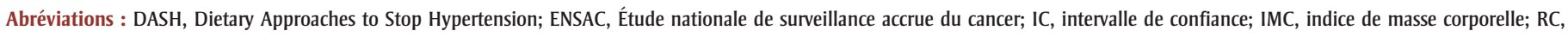
rapport de cotes; Réf., référence.

Remarque : Les totaux peuvent varier du fait de valeurs manquantes.

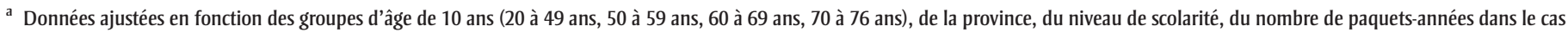
des fumeurs, de la pratique d'une activité physique intense ou modérée, de la prise de suppléments de calcium et de l'âge à la première grossesse.

mais la différence entre les cas et les témoins n'était pas statistiquement significative. Nous n'avons pas recueilli de données sur l'hormonothérapie substitutive (HTS) ou la prise de contraceptifs oraux (CO), malgré l'existence d'une relation entre ces variables et le risque de CCR. L'HTS est inversement liée au risque de CCR dans la plupart des études, notamment dans la Women's Health Initiative, où une diminution de $36 \%$ du risque de CCR a été observée chez les femmes recevant une HTS $^{39-41}$. Le groupe d'âge prédominant quant à l'usage de l'HTS est celui des 50 à 69 ans. Dans notre étude, 63 \% des cas et $53 \%$ des témoins faisaient partie de ce groupe d'âge. Au moment de l'étude, le recours à l'HTS était au maximum à environ $40 \%$ chez les Canadiennes de 50 à 59 ans et à près de $20 \%$ chez les Canadiennes de 60 à 69 ans $^{42}$. Il est donc possible que l'HTS ait joué un rôle protecteur chez une forte proportion de participantes. Néanmoins, selon une autre étude où l'on avait tenu compte de l'HTS dans la modélisation logistique, le régime DASH n’a été associé à aucun effet statistiquement significatif chez les femmes alors qu'il l'était chez les hommes ${ }^{11}$. Chez les femmes plus jeunes, la prise de CO peut avoir atténué les effets d'un profil DASH faible, car certaines études ${ }^{43,44}$ ont révélé une relation inverse entre les CO et le risque de CCR chez les femmes ayant déjà pris ou prenant des CO. Cependant, nous soupçonnons que l'influence potentielle des CO sur le risque de CCR est négligeable.

Nos observations selon lesquelles un profil DASH fort est associé à un risque réduit de CCR chez les hommes concordent avec les données montrant qu'il y aurait une relation entre certains facteurs alimentaires et le CCR. Dans une analyse portant sur l'alimentation et la prévention du cancer à l'échelle mondiale ${ }^{10}$, l'ensemble des catégories alimentaires énumérées dans notre étude (ou les principaux nutriments dont elles sont composées), à l'exception du sodium, figurait sur une liste de facteurs jouant un rôle plus ou moins important dans l'apparition du CCR. Il s'agissait notamment des aliments riches en fibres alimentaires (p. ex. les légumineuses), des légumes, des fruits, de la viande, du lait et des aliments riches en vitamine $\mathrm{D}$ ou en calcium, des sucres, de l'alcool, des graisses saturées et des aliments riches en sélénium, comme les noix, les graines et les céréales entières. Cette analyse menée à l'échelle mondiale nous fournit un cadre scientifique à partir duquel explorer le régime DASH pour étudier le risque de CCR. L'analyse nous permet également de démontrer la plausibilité biologique de l'association inverse que nous avons 
observée entre un score DASH élevé et le risque de CCR chez les hommes.

Les différences observées entre les cas et les témoins quant à l'apport en certains éléments du régime DASH variaient en fonction du sexe. Certains éléments pourraient avoir joué un rôle plus déterminant que d'autres. Chez les cas de sexe masculin, on a observé une consommation supérieure de graisses saturées, d'alcool et de sucreries (nutriments dits négatifs). Une telle tendance associée à des nutriments dits négatifs n'était pas apparente chez les femmes. Chez les cas de sexe féminin, on a observé une consommation supérieure de fruits et de céréales entières (nutriments dits positifs), ce qui donne à penser que des facteurs annulant l'effet positif de ces éléments pourraient être en cause. Ces résultats concordent avec les observations faites par d'autres chercheurs, selon lesquelles la consommation de grandes quantités d'alcool (et de viande et de céréales transformées) était associée à un risque accru de CCR, risque par ailleurs atténué lorsqu'on consommait davantage de fruits, de légumes et de céréales entières ${ }^{4}$.

\section{Limites}

S'agissant d'une étude cas-témoins, un biais de rappel, ainsi que les faiblesses qui en découlent, est inévitable. Cela est particulièrement vrai lorsqu'il est question de se rappeler son alimentation sur une période de 24 mois.

L'évaluation des habitudes alimentaires comporte un certain degré de subjectivitée $^{4,11,45}$, et c'est également vrai pour la façon dont les auteurs définissent et évaluent l'observance d'un régime $\mathrm{DASH}^{28,46-48}$. Dans notre étude, nous nous sommes fondés sur les données disponibles pour définir les groupes alimentaires, répartir les aliments pertinents dans chacun de ces groupes et déterminer les portions équivalentes. Ainsi, il est possible que nous ayons mal classé certains aliments, ce qui pourrait avoir influé sur le score DASH des participants et avoir entraîné une surreprésentation des scores médians. Les scores médians sont difficiles à interpréter parce qu'ils peuvent être dus à un manque d'attributs positifs, à la présence de nombreux attributs négatifs ou à une association de ces deux facteurs. En ce qui concerne le fait que peu de participants aient eu un score DASH élevé dans notre étude, il est à noter que la même observation a été faite dans une étude semblable à la nôtre ${ }^{11}$. De plus, le questionnaire que nous avons utilisé était une version abrégée des questionnaires de Block et de Willett et ne comportait que 69 éléments. Si on le compare à d'autres questionnaires du même type ${ }^{11,12}$, il est possible que notre questionnaire ait été trop limité pour bien rendre compte de l'ensemble des aliments associés au régime DASH.

Nous avons par ailleurs accordé une pondération égale aux 10 groupes alimentaires pour calculer le score DASH, mais il est probable les catégories alimentaires n'ont pas toutes le même effet sur le $\mathrm{CCR}^{29}$. Par exemple, les viandes rouges et transformées sont associées de façon probante à un risque accru de CCR, mais les graisses saturées le sont moins ${ }^{10}$. Les différences observées entre les sexes pourraient signifier qu'il serait important d'accorder une pondération différente à certains aliments, et en particulier en fonction du sexe des sujets. Par exemple, l'alcool est associé de façon probante au CCR chez les hommes, mais une telle association n'est que probable chez les femmes ${ }^{10}$.

\section{Conclusion}

Nos observations donnent à penser que le régime $\mathrm{DASH}$ serait associé à un risque inférieur de CCR, en particulier chez les hommes. Des études ultérieures pourraient examiner les différences observées entre les sexes et évaluer l'importance éventuelle du régime DASH au-delà de la prévention du CCR.

\section{Références}

1. Société canadienne du cancer. Statistiques canadiennes sur le cancer 2013 [Internet]. Ottawa (Ont.) : Société canadienne du cancer; 2013. Consultable en ligne à partir de la page : http://www.cancer.ca/fr-ca /cancer-information/cancer-101/canadian -cancer-statistics-publication/?region = onhttp: //www.cancer.ca/en/cancer-information /cancer-101/canadian-cancer-statistics -publication/?region $=$ on
2. Agence de la santé publique du Canada. Cancer colorectal, Facteurs de risque [Internet]. Ottawa (Ont.) : Agence de la santé publique du Canada; 2013 [Consultation le 16 décembre 2013]. Consultable en ligne à la page : http://www.phac-aspc.gc.ca/cd-mc /cancer/cancer_colorectal-colorectal_cancer -fra.phphttp://www.phac-aspc.gc.ca/cd-mc /cancer/colorectal_cancer-cancer_colorectal -eng.php

3. Huxley RR, Ansary-Moghaddam A, Clifton P, Czernichow S, Parr CL, Woodward M. The impact of dietary and lifestyle risk factors on risk of colorectal cancer: a quantitative overview of the epidemiological evidence. Int J Cancer. 2009;125(1):171180.

4. Randi G, Edefonti V, Ferraroni M, La Vecchia C, Decarli A. Dietary patterns and the risk of colorectal cancer and adenomas. Nutr Rev. 2010;68:389-408.

5. Harnden KE, Frayn KN, Hodson L. Dietary Approaches to Stop Hypertension (DASH) diet: applicability and acceptability to a UK population. J Hum Nutr Diet. 2010;23:3-10.

6. Appel LJ, Moore TJ, Obarzanek E, et al. A clinical trial of the effects of dietary patterns on blood pressure. N Engl J Med. 1997;336: 1117-1124.

7. Taylor EN, Stampfer MJ, Mount DB, Curhan GC. DASH-style diet and 24-hour urine composition. Clin J Am Soc Nephrol. 2010; 5(12):2315-22. doi: 10.2215/CJN.04420510

8. Azadbakht L, Mirmiran P, Esmaillzadeh A, Azizi T, Azizi F. Beneficial effects of a Dietary Approaches to Stop Hypertension eating plan on features of the metabolic syndrome. Diabetes Care. 2005;28:28232831.

9. Tobias DK, Zhang C, Chavarro J et collab. Prepregnancy adherence to dietary patterns and lower risk of gestational diabetes mellitus. Am J Clin Nutr. 2012;96:289-295.

10. Fonds Mondial de Recherche contre le Cancer/American Institute for Cancer Research. Alimentation, Nutrition, Activité Physique et Prévention du Cancer : une Perspective Mondiale Washington (DC) : AICR; 2007. 
11. Dixon LB, Subar AF, Peters U et collab. Adherence to the USDA food guide, DASH eating plan, and Mediterranean dietary pattern reduces risk of colorectal adenoma. J Nutr. 2007;137:2443-2450.

12. Fung TT, Hu FB, Chiuve SE, Fuchs CS, Giovannucci E. The Mediterranean and Dietary Approaches to Stop Hypertension (DASH) diets and colorectal cancer. Am J Clin Nutr. 2010;92:1429-1435.

13. Mekary RA, Hu FB, Willett WC et collab. The joint association of eating frequency and diet quality with colorectal cancer risk in the Health Professionals Follow-up Study. Am J Epidemiol. 2012;175:664-672.

14. Statistique Canada. Hypertension artérielle, 2011 [Internet]. Ottawa (Ont.) : Statistique Canada; 2013 [consultation le 16 décembre 2013]. Consultable en ligne à la page : http://www.statcan.gc.ca/pub/82-625-x /2012001/article/11663-fra.htm

15. Villeneuve PJ, Johnson KC, Kreiger N, Mao Y. Risk factors for prostate cancer: results from the Canadian National Enhanced Cancer Surveillance System. The Canadian Cancer Registries Epidemiology Research Group. Cancer Causes Control. 1999;10:355367.

16. Frise S, Kreiger N, Gallinger S, Tomlinson G, Cotterchio M. Menstrual and reproductive risk factors and risk for gastric adenocarcinoma in women: findings from the Canadian National Enhanced Cancer Surveillance System. Ann Epidemiol. 2006;16:908-916.

17. Hu J, La Vecchia C, Negri E, Mery L. Nutrients and risk of colon cancer. Cancers. 2010;2:51-76.

18. Percy C, Holten VV, Muir C (dir.). International classification of diseases for oncology, $2^{\mathrm{e}}$ éd. Genève $(\mathrm{CH})$ : Organisation mondiale de la santé; 1990.

19. Johnson KC, Mao Y, Argo J, Dubois S, Semenciw R, Lava Jl. The National Enhanced Cancer Surveillance System: a case-control approach to environmentrelated cancer surveillance in Canada. Environmetrics. 1998;9:495-504.
20. Pan SY, Desmeules M, Morrison H, Wen SW, and the Canadian Cancer Registries Epidemiology Research Group. Obesity, high energy intake, lack of physical activity, and the risk of kidney cancer. Cancer Epidemiol Biomarkers Prev. 2006;15:24532460.

21. Organisation mondiale de la Santé. Obésité : prévention et prise en charge de l'épidémie mondiale. Rapport d'une consultation de l'OMS. Genève (Suisse) : Organisation mondiale de la Santé; 2000.

22. Block G, Hartman AM, Naughton D. A reduced dietary questionnaire: development and validation. Epidemiology. 1990;1:58-64.

23. Willett WC. Nutritional epidemiology, $2^{\mathrm{e}}$ éd. New York (NY) : Oxford University Press; 1998.

24. Santé Canada. Fichier canadien sur les éléments nutritifs : recueil des données canadiennes de composition des aliments. Guide d'utilisation. Ottawa (Ont.) : Santé Canada; 2005.

25. Mitrou PN, Kipnis V, Thiebaut AC, et al. Mediterranean dietary pattern and prediction of all-cause mortality in a US population. Arch Intern Med. 2007;167:2461-2468.

26. U.S. Department of Health and Human Services. Dietary guidelines for Americans, 2005. Appendix A. Eating patterns. Appendix A-1: The DASH eating plan at 1,600-, 2,000-, 2,600-, and 3,100-calorie levels [Internet]. Rockville (MD): U.S. Department of Health and Human Services; 2012 [consultation le 13 février 2012]. Consultable en ligne à la page : http:// www.health.gov/dietaryguidelines/dga2005 /document/html/appendixa.htmhttp://www .health.gov/dietaryguidelines/dga2005 /document/html/appendixa.htm

27. SAS Institute Inc. The SAS system for Windows release 9.01. Carey (NC) : SAS Institute Inc.; 2002.

28. Fung TT, Chiuve SE, McCullough ML, Rexrode KM, Logroscino G, Hu FB. Adherence to a DASH-style diet and risk of coronary heart disease and stroke in women. Arch Intern Med. 2008;168:713720.
29. Reedy J, Mitrou PN, Krebs-Smith SM et collab. Index-based dietary patterns risk of colorectal cancer. The NIH-AARP Diet and Study. Am J Epidemiol. 2008;168:38-48.

30. Bedard A, Riverin M, Dodin S, Corneau L, Lemieux S. Sex difference in the impact of the Mediterranean diet on cardiovascular risk profile. Br J Nutr. 2012;108:1428-1434.

31. Carter SJ, Roberts MB, Salter J, Eaton CB. Relationship between Mediterranean diet score and atherothrombotic risk: findings from the third National Health and Nutrition Examination Survey (NHANES III), 19881994. Atherosclerosis. 2010;210:630-636.

32. Gribovskaja-Rupp I, Kosinski L, Ludwig KA. Obesity and colorectal cancer. Clin Colon Rectal Surg. 2011;24:229-243.

33. Buysschaert M, Sadikot S. Diabetes and cancer: a 2013 synopsis. Diabetes Metab Syndr. 2013;7:247-250.

34. Larsson SC, Orsini N, Wolk A. Diabetes mellitus and risk of colorectal cancer: a meta-analysis. J Natl Cancer Inst. 2005;97: 1679-1687.

35. Fung TT, Hu FB, Schulze $M$ et collab. A dietary pattern that is associated with $\mathrm{C}$ peptide and risk of colorectal cancer in women. Cancer Causes Control. 2012;23: 959-965.

36. Wernli KJ, Wang Y, Zheng Y, Potter JD, Newcomb PA. The relationship between gravidity and parity and colorectal cancer risk. J Womens Health. 2009;18:995-1001.

37. Zervoudakis A, Strickler HD, Park Y et collab. Reproductive history and risk of colorectal cancer risk in postmenopausal women. J Natl Cancer Inst. 2011;103:826834.

38. Nichols HB, Trentham-Dietz A, Hampton JM, Newcomb PA. Oral contraceptive use, reproductive factors, and colorectal cancer risk: findings from Wisconsin. Cancer Epidemiol Biomarkers Prev. 2005;14:1212-1218.

39. Rossouw JE, Anderson GL, Prentice RL et collab. Risks and benefits of estrogen plus progestin in healthy postmenopausal women; principal results from the Women's Health Initiative. JAMA. 2002;288:321-333. 
40. Kampman E, Bijl AJ, Kok C, van’t Veer P. Reproductive and hormonal factors in male and female colon cancer. Eur J Cancer Prev. 1994;3:329-336.

41. Lin PH, Allen JD, Li YJ, Yu M, Lien LF, Svetkey LP. Blood pressure-lowering mechanisms of the DASH dietary pattern. J Nutr Metab. 2012;2012:472396. doi: $10.1155 / 2012 / 472396$.

42. De P, Neutel CI, Olivotto I, Morrison H. Breast cancer incidence and hormone replacement therapy in Canada. J Natl Cancer Inst. 2010;102:1489-1495.

43. Lin J, Zhang SM, Cook NR, Manson JE, Buring JE, Lee IM. Oral contraceptives, reproductive factors, and risk of colorectal cancer among women in a prospective cohort study. Am J Epidemiol. 2007;165:794-801.

44. Martinez ME, Grodstein F, Giovannucci E et collab. A prospective study of reproductive factors, oral contraceptive use, and risk of colorectal cancer. Cancer Epidemiol Biomarkers Prev. 1997;6:1-5.

45. Jones-McLean EM, Shatenstein B, Whiting SJ. Dietary pattern research and its application to nutrition policy for the prevention of chronic disease among diverse North American populations. Appl Physiol Nutr Metab. 2010;35:195-198.

46. Hajna S, Liu J, LeBlanc P, Faught BE et collab. Association between body composition and conformity to the recommendations of Canada's Food Guide and the Dietary Approaches to Stop Hypertension (DASH) diet in peri-adolescence. Public Health Nutr. 2012;15:1890-1896.

47. Liese AD, Nichols $M$, Sun X, D’Agostino $\mathrm{RB}$, Haffner SM. Adherence to the DASH diet is inversely associated with incidence of type 2 diabetes: the Insulin Resistance Atherosclerosis Study. Diabetes Care. 2009;32:1434-1436.

48. table-5-captionWhitt-Glover MC, Hunter JC, Foy CG et collab. Translating the Dietary Approaches to Stop Hypertension (DASH) diet for use in underresourced, urban African American communities, 2010. Prev Chronic Dis. 2013;10:120088. doi: $10.5888 /$ pcd 10.120088 